\title{
Software para administrar recetas de comida típica de la región
}

\section{Software to manage recipes of typical food of the region}

\author{
BETANCOURT-SÁNCHEZ, Ricardo Gabino†*, LEVARIO-TORRES, José Guadalupe, \\ VILLANUEVA-FLORES, Juan Antonio y GALLEGOS-HERRERA, Juan Manuel
}

Instituto Tecnológico Superior de Santiago Papasquiaro. Km. 114 Carr. J. Gpe. Aguilera-Guanaceví, Santiago Papasquiaro, Durango

ID $1^{\text {er }}$ Autor: Ricardo Gabino, Betancourt-Sánchez, / ORC ID: 0000-0003-3917-2793, Researcher ID Thomson: S-27802018, arXiv Author ID: beta20991, PubMed Autor ID: beta20991, CVU CONACYT ID: 260735

ID $1^{\text {er }}$ Coautor: José Guadalupe, Levario-Torres / ORC ID: 0000-0002-0576-6908, Researcher ID Thomson: S-2869-2018arXiv Author ID: levariot, PubMed Autor ID: levariot, CVU CONACYT ID: 260831

ID $2^{\text {do }}$ Coautor: Juan Antonio, Villanueva-Flores / ORC ID: 0000-0002-6825-2509, arXiv Author ID: ccnajavf, CVU CONACYT ID: 614407

ID $3^{\text {er }}$ Coautor: Juan Manuel, Gallegos-Herrera / ORC ID: 0000-0003-3629-1930, arXiv Author ID: JMGallegos, CVU CONACYT ID: 614663

DOI: $10.35429 / J C T .2019 .11 .3 .23 .31$

Recibido 30 de Octubre, 2019, Aceptado, 07 de Diciembre, 2019

\begin{abstract}
Resumen
Desarrollo de un software que permite facilitar la administración de recetas de comida típicas de la región, mismas que se pueden compartir con otros usuarios, además de poder darle seguimiento a la información subida en la web de forma rápida y sencilla. Se busca que con el uso de un software de este tipo, los usuarios que tengan acceso a la plataforma puedan compartir y de la misma forma tomar, recetas típicas de la región y de otras regiones, para preservar las tradiciones y tener una variedad de información con lo que tendrían una diversidad de posibilidades al preparar comidas. Por otra parte, también se obtuvo un prototipo funcional del software, el cual se logro siguiendo una metodología de desarrollo en espiral
\end{abstract}

Software, Administración de información, Metodología de desarrollo en espiral, recetas

\begin{abstract}
Development of software that facilitates the administration of typical food recipes from the region, which can be shared with other users, in addition to being able to follow up the information posted on the web quickly and easily. It is sought that with the use of software of this type, users who have access to the platform can share and in the same way take, typical recipes from the region and from other regions, to preserve traditions and have a variety of information with which they would have a diversity of possibilities when preparing meals. On the other hand, a functional prototype of the software was also obtained, which was achieved following a spiral development methodology.
\end{abstract}

Software, Information management, Spiral development methodology, Recipes

\footnotetext{
* Correspondencia del Autor (Correo electrónico: beta20991@ gmail.com)

$\dagger$ Investigador contribuyendo como primer autor.
} 


\section{Introducción}

En el presente trabajo se visualiza hacia la necesidad de darle a conocer a todas las personas, sobre la gastronomía y las comidas tradicionales de distintos lugares, esto pues para rescatar y preservarlas en beneficio de las próximas generaciones.

Con la finalidad de que las recetas de culturas distintas se compartan en un ámbito en el cual no se pierdan y estas puedan persistir en la sociedad actual.

Dicha razón impulsa a dar a conocer la gastronomía de distintos lugares para que estas se conserven y evitar las consecuencias como que se pierdan por nuevas costumbres. El resultado de esta investigación dará uno de los primeros proyectos que se ha recogido sobre las recetas que aspira ayudar a mantener viva tradiciones, costumbres y riqueza gastronómica, para que de esta manera se pueda incentivar en los jóvenes su consumo, producción y difusión, como parte de la herencia cultural que obligatoriamente debemos legar a las futuras generaciones.

Todo esto para que las nuevas generaciones, como lo son los jóvenes, puedan saber cómo se desarrollaba la gastronomía en tiempos pasado y los pasos de la elaboración que llevaba.

Incluso existen recetas que la gente tiene personales, son recetas que no son específicamente conocidas ante la sociedad, se pudiera decir que son recetas de familia, por lo tanto, se pueden preservar a través de los recetarios e inclusive se pueden publicar en distintos ámbitos para que las demás personas puedan realizarlas y así que se dé a conocer ante más sociedad.

Según Pressman, R. S. (2010), la era del conocimiento en que vivimos no sólo está cambiando la sociedad en sí misma, sino que los nuevos modelos de negocios requieren la reformulación de nuevos conceptos. Conocimiento, activos intangibles, desarrollo de software, Web, etc., son algunos de los términos más utilizados en cualquier ambiente $\mathrm{o}$ negociación. Esta era del conocimiento requiere de nuevas tendencias apoyadas precisamente en el conocimiento.
Como lo comenta Ian Sommerville (2011) la ingeniería del software no es una excepción, y por ello se requiere no sólo una actualización de conceptos, sino también una comprensión y una formulación del nuevo conocimiento existente en torno a las nuevas innovaciones y teorías de dicha disciplina.

En estos tiempos el uso de dispositivos electrónicos como celulares y PCs son herramientas indispensables para el desarrollo de la vida en la actualidad, más sin embargo estos dispositivos electrónicos dependen de su funcionamiento del uso del software.

Lo anterior explica el cómo actualmente muchas de las decisiones que se toman en la vida se basan en los resultados y recomendaciones resultantes del software.

De una forma tradicional un recetario es el libro que recopila diversas recetas de cocina. Estas recetas consisten en la descripción de los pasos a seguir para preparar una comida, incluyendo también los ingredientes que se necesitan.

Son los pasos que se transcriben para realizar mil variedades de platillos de diferentes culturas, distintos métodos, ingredientes, etc.

Es frecuente que las recetas de cocina se transmitan a través de la vía oral, pasando de generación en generación. Sin embargo, al ser recopilada en un recetario, su alcance se multiplica.

Se comenta que una receta registrada en un recetario permite, por otra parte, que una preparación gastronómica permanezca en el tiempo, ya que sus ingredientes y elaboración han quedado consignados.

En algunos casos, los recetarios se crean en torno a un electrodoméstico de cocina, como ocurrió en su momento con el microondas, para facilitar a los nuevos clientes la comprensión de su funcionamiento y las posibilidades que le ofrecía.

La visión de la movilidad empresarial y de servicios está siendo tomada en cuenta por grandes corporaciones, las que invierten un gran capital en el desarrollo de componentes electrónicos y de software.

BETANCOURT-SÁNCHEZ, Ricardo Gabino, LEVARIOTORRES, José Guadalupe, VILLANUEVA-FLORES, Juan Antonio y GALLEGOS-HERRERA, Juan Manuel. Software para administrar recetas de comida típica de la región. Revista de Tecnología Informática. 2019 
Por otro lado, a las universidades e instituciones les corresponde generar el recurso humano capaz de implementar, innovar y desarrollar nueva tecnología dirigida a aplicaciones como el trabajo colaborativo, la automatización y el control industrial, la educación remota y el entretenimiento, entre otras.

Si una receta se obtuviera oralmente, las personas en ocasiones no logran recordar todos los ingredientes o los pasos a seguir para su elaboración, provocando así una alteración de la receta, la cual no va a ser la misma desde ese momento, porque después se van a quedar con esa idea las siguientes generaciones.

De aquí la importancia de registrar las recetas, o de tener pruebas de que son propias, ya que muchas personas suelen apoderarse de algunas por no tener un registro. Los recetarios pueden hacerse de distintas comidas, culturas, procesos, ingredientes, etc.

Otro de los problemas es que muchos de los recetarios que existen se difunden de manera impresa o no de una manera digial, y la mayoria de las veces no son recetas tipicas de la región noroeste de nuestro estado y pais, siendo que estas son diseñadas por personas de diferentes regiones.

Para ayudar a mitigar el problema, es que el software ayudaría a guardar y distribuir entre los usuarios las diferentes recetas que puedan aportar los usuarios.

Con la utilización de esta aplicación, se bridará un apoyo a las personas que realizan labores de cocina, ya que brindará la información necesaria para conocer y comprender algunas recetas nuevas, igualmente brinda la oportunidad de almacenar las recetas propias de cada usuario, compartirla con otras personas que les guste las actividades gastronómicas y por ello, conservar la cultura.

Esta aplicación es una opción de poder tener un control de las recetas propias de cada persona, ya que cada persona tiene su estilo y manera de realizarla.

Para el público joven, la aplicación ayuda a poder aprender recetas nuevas, a reforzar las recetas que ya conocemos, igualmente ayuda a poder compartirlas de una manera fácil y clara.
También nos permite crear una base de almacenamiento de la información propia del usuario, al igual que almacenar las recetas ya antes introducidas.

Para las amas de casa que son el principal público al que va dirigida la aplicación, ya que son, por lo general, las que realmente podría ser útil esta aplicación, puesto que ayuda a organizar y administrar las recetas, ya sean personales, o que se quieren compartir con las personas que también tengan la aplicación, permitiendo así, la conservación de las tradiciones familiares, permitiendo conocer distintas comidas de lugares distintos, lo cual da lugar a una diversidad cultural mucho más rica.

A continuación se presenta como primer apartado el método que se utilizo para el desarrollo del software y la relación de este con las personas, sus costubres y lo relacionado a ello.

En el segundo apartado se muestra la descripción de la constitución del software y las funciones que desempeñan cada uno de ellos.

Existe un apartado de resultados el cual muestra algunas de las pantallas más importantes que conforman el software, y por último se incluye el apartado de conclusiones.

\section{Descripción del método y la relación del software con las personas $y$ sus costumbres}

Según Eric Braude (2003) las tecnologías de la información son "aquellas herramientas y métodos empleados para recabar, retener, manipular o distribuir información. La tecnología de la información se encuentra generalmente asociada con las computadoras y las tecnologías afines aplicadas a la toma de decisiones". Como menciona Piattini (2007) las TIC ofrecen nuevos entornos y nuevas posibilidades, se suman a otros recursos ya conocidos como el correo electrónico, mensajería instantánea o chat, foros de discusión plataformas educativas, simuladores, materiales multimedia, etc.

Para el desarrollo del software, se utilizó como base el Modelo de Proceso Evolutivo (Espiral).

BETANCOURT-SÁNCHEZ, Ricardo Gabino, LEVARIOTORRES, José Guadalupe, VILLANUEVA-FLORES, Juan Antonio y GALLEGOS-HERRERA, Juan Manuel. Software para administrar recetas de comida típica de la región. Revista de Tecnología Informática. 2019 
El cual consta de un número de actividades estructurales, también llamadas "regiones de tareas", que a continuación se describen y se denotan en la Figura 1.

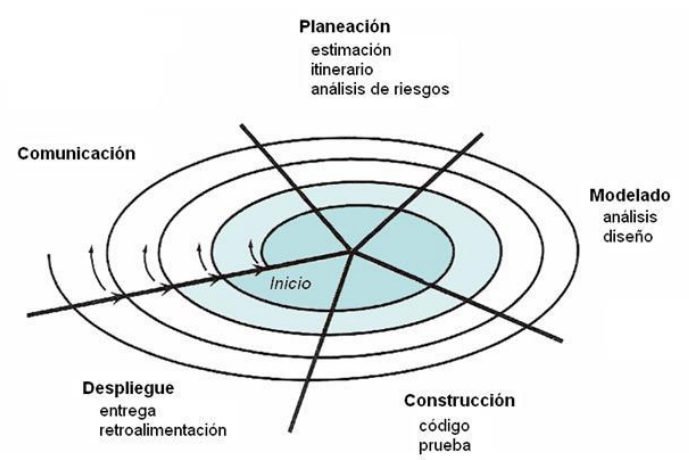

Figura 1 Modelo en Espiral

Comunicación con el usuario.- Define las tareas requeridas para establecer comunicación entre el coordinador del desarrollo del software, para revisar especificaciones y plantear necesidades del software.

Planeación.- Define las tareas gestión de recursos, tiempos e información relacionada con el software.

Modelado.- Define las tareas para construir una o más representaciones del software.

Construcción.- Define las tareas para construir, probar e implementar el software.

Despliegue.- Define las tareas de evaluación de las representaciones del software creada durante el modelado y su construcción.

Cuando empieza este proceso evolutivo, el equipo de trabajo gira alrededor de las agujas del reloj, comenzando por el centro. El primer circuito de la espiral produce el desarrollo de una especificación de productos, los pasos siguientes en la espiral se podrían utilizar para desarrollar un prototipo y progresivamente versiones más sofisticadas del software. Cada paso de la región de planificación produce ajustes en el plan del software.

La planificación se ajusta en función de la evaluación del software. Además, el administrador del proyecto ajusta el número planificado de iteraciones requeridas para completar el desarrollo del software.
La simulación, es una de las herramientas más ampliamente utilizadas en la actualidad, debido a una estructura fácil y rápida de comprender. Además de la disponibilidad de lenguajes de propósito especial existentes hoy en día. La simulación, es la imitación de la operación de un proceso o sistema del mundo real, a través del tiempo. Involucra la generación de una historia virtual del sistema, y su observación, y esto ayuda a tener inferencias concernientes a las características de operación del sistema real.

Desde el inicio se determinó los roles que cada integrante del equipo debería seguir, y las formas de comunicar los avances, horarios, resultados, problemas presentados, y la forma de entregar resultados de cada fase. En la parte de planeación, se analizaron los riesgos, resultados esperados, la estimación de tiempos de cada una de las fases, posibles traslapes, adelantos en el desarrollo y tiempos de adecuaciones y pruebas. Se consideraron además las fases que se muestran en la Tabla 1.

\begin{tabular}{|c|l|}
\hline 1 & Preparación e integración del equipo del proyecto \\
\hline 2 & Formulación de la especificación requerimientos \\
\hline 3 & Análisis de la base de datos \\
\hline 4 & Análisis y diseño preliminar del software \\
\hline 5 & Desarrollo del software \\
\hline 6 & Creación del manual de usuario \\
\hline 7 & Creación del manual de instalación \\
\hline 8 & Integración de la documentación y el software generado \\
\hline 9 & Implementación del software para pruebas \\
\hline 10 & Realizar pruebas al software \\
\hline 11 & Generar documentación de las pruebas \\
\hline 12 & Realizar correcciones al software \\
\hline 13 & Implementación final del software \\
\hline
\end{tabular}

Tabla 1 Fases del desarrollo del software

En nuestra sociedad, el envejecimiento de la población es un hecho palpable. Una vez que se están viendo cubiertas las necesidades básicas o primarias, atentos a las necesidades de un sociedad del bienestar, hay que atender a este creciente sector con diversos programas sociales, culturales educativos y sanitarios, que intentan dar respuesta las nuevas necesidades, pero también para adaptarlos para que entren con la sociedad en la nueva era.

Uniendo dos cuestiones básicas, facilitarles la formación y el acceso a los nuevos medios de información y comunicación, al tiempo que fomentar en ellos su interdependencia, comunicación y autonomía como para se puedan conducir dignamente por la sociedad que empieza a irrumpir.

BETANCOURT-SÁNCHEZ, Ricardo Gabino, LEVARIOTORRES, José Guadalupe, VILLANUEVA-FLORES, Juan Antonio y GALLEGOS-HERRERA, Juan Manuel. Software para administrar recetas de comida típica de la región. Revista de Tecnología Informática. 2019 
¿Qué puede aportar el uso de tic's a las personas mayores?

Mejorar la relación intergeneracional: en la actualidad, el mayor, al dejar su actividad laboral, deja también gran parte de su valiosa aportación a la sociedad. Afortunadamente comienza a emerger una nueva filosofía de educación de los mayores en la que se intenta que en una sociedad de objetivos productivos, cuando el anciano sale del ámbito laboral, no salga también de la consideración social, puesto que se desaprovecharía un cúmulo de conocimientos y sabiduría. Creemos que con las nuevas tecnologías se puede promover el principio de solidaridad entre generaciones fomentar el voluntariado de las personas mayores hacia los jóvenes a la hora de transmitirles sus conocimientos y experiencias profesionales y de otro tipo.

Según el Instituto de Estudios Superiores en Gastronomía, la historia de cada uno de los pueblos se presenta en su cultura y en sus formas de alimentarse, ya que son partes esenciales de ella, porque consigo lleva costumbres, producción de sus suelos, interrelaciones de culturas, por las migraciones que nos permite conocer claramente cómo vivieron sus pueblos, y hacia donde nos proyectamos, por el cambio de costumbres y sus consecuencias.

\section{Descripción del Software}

El software del sitio web será una aplicación que mostrará todas las recetas de comida subida por diferentes usuarios previamente registrados en la página, el visitante podrá ver todas las recetas subidas, buscar una receta o registrar un perfil para subir sus propias recetas, además de que la pagina muestra diferentes secciones donde clasifica los diferentes tipos de recetas existentes en la base de datos de la aplicación.

Para la creación de este software fuen necesario el hacer un análisis del personal involucrado, las prespectivas del producto, ya que desde este punto de vista el software no depende de ningun otro sistema mayor. Dentro de las funciones para cada tipo de usuario se definieron las siguientes:

\section{Usuario}

\section{- Ver las recetas \\ - Comentar las recetas}

- Calificar las recetas

- $\quad$ Registrar un perfil

\section{Usuarios con cuenta}

\section{- $\quad$ Subir recetas \\ - Modificar recetas \\ - $\quad$ Ver su historial de recetas}

Tambien se definieron las caracteristicas de los usuarios, tanto para los que son administradores, como los que son usuarios estandar, se consideraron restricciones, supociones y dependencia, así como la evolución previsible del sistema.

En la Figura 2 se muestra el diagrama del mapa del sitio y la organización de la aplicación web del recetario.

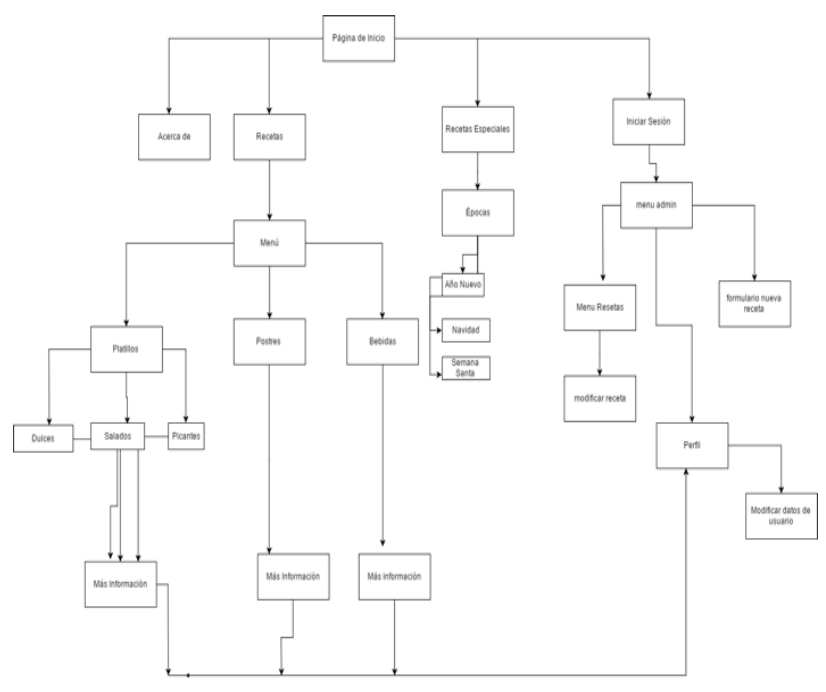

Figura 2 Diagrama del mapa del sitio Fuente: Elaboración Propia

En la tabla 2 se muestra un ejemplo de los requisitos específicos que debieron considerarse.

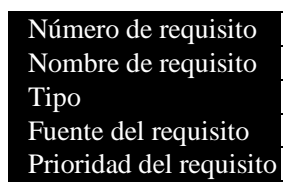

RF1

Inicio de sesión

X Requisito $\square$ Restricción

Entrevista al cliente

X Alta/Escencial $\square$ Media/deseado $\square$ Baja/ opcional

Número de requisito
Nombre de requisito

Nombre de requisito

Tipo

Fuente del requisito

RF2
Registro de usuarios

$X$ Requisito $\square$ Restricción

Entrevista al cliente

X Alta/Escencial $\square$ Media/deseado $\square$ Baja/ opcional

Tabla 2 Ejemplo de requisitos específicos Fuente: Elaboración Propia

Dentro de los requisitos comunes que se debieron considerar, estan los de las interfaces:

BETANCOURT-SÁNCHEZ, Ricardo Gabino, LEVARIOTORRES, José Guadalupe, VILLANUEVA-FLORES, Juan Antonio y GALLEGOS-HERRERA, Juan Manuel. Software para administrar recetas de comida típica de la región. Revista de Tecnología Informática. 2019 
Usuario: Los usuarios generales tendrán acceso a ver todas las recetas que estén registradas previamente, podrá realizar búsquedas de alguna receta o usuario favorito, podrá escribir un comentario en ellas y calificar según su criterio, también los usuarios contarán con la opción de registrarse como usuario privilegiado.

Usuario privilegiado: los usuarios privilegiados contarán con un perfil en el que podrán editar datos como nombre, foto, etc. Tendrá acceso a todas las recetas que haya registrado, las podrá editar y eliminar.

Dentro de lo anterior, se tuvo que pensar en las interfaces de usuario, las interfaces de hardware, interfaces de software e interfaces de comunicación. Por otra parte fue necesario el definir requisitos funcionales, dentro de los cuales algunos ejemplos de ellos fueron:

- $\quad$ La autentificación de los usuarios.

- Poder consultar información, tanto de los usuarios como de las recetas.

- $\quad$ El poder registrar usuarios y recetas.

- El hacer modificaciones de usuarios como de recetas.

- $\quad$ Y el de eliminacion de información.

Dentro de los requisitos no funcionales fuen necesario el considerar los requisitos de rendimiento, de seguridad, de fiabilidad, disponibilidad, mantenibilidad y portabilidad, así como cualquier otro requisito no previsto. Cuando se llegó a la hora de la generación del código, fue necesario la parte de las generaciones de:

\section{- $\quad$ Código (Modelo). \\ - Código Vista. \\ - $\quad$ Estilos CSS.}

\section{Resultados}

Una vez que termino la fase de programación del software, se pasó a la parte de pruebas y adecuaciones del mismo, mismas en las que se documentaron los errores para su posterior corrección. También traslapado dentro de estas partes, se comenzó con la documentación de todo el software, así como los procedimientos de instalación, manejador de la base de datos y puesta a punto de las configuraciones.
Como resultado, se logró obtener un prototipo funcional del software para ser puesto a prueba y con esto evaluar su funcionalidad y calidad.

Durante las pruebas realizadas, el software logro de manera correcta la función para la que fue creado, dando pie a que sea puesto en marcha en situaciones reales y buscando que logre la satisfacción de los usuarios, tanto administradores, clientes y cocineros.

En la Imagen 3 se muestra el logotipo que identifica la aplicación web del recetario.

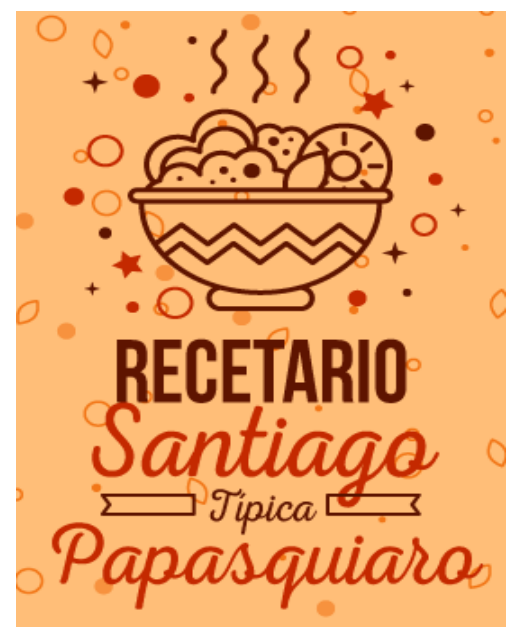

Figura 3 Logotipo del recetario

Fuente: Elaboración Propia

$\mathrm{Al}$ momento de acceder a la página web se encontrará con la página de inicio donde podrá ver todo acerca del recetario, podrá interactuar con todos los menús y visualizar todo lo que contiene, mas no podrá subir ni modificar nada hasta no estar registrado como un usuario.

En la Imagen 4 se muestra como en el inicio del software encontrar información acerca de platillos, sopas, bebidas, postres, repostería, salsas, cada uno contará con un apartado distinto donde podrá elegir la opción que más le guste.

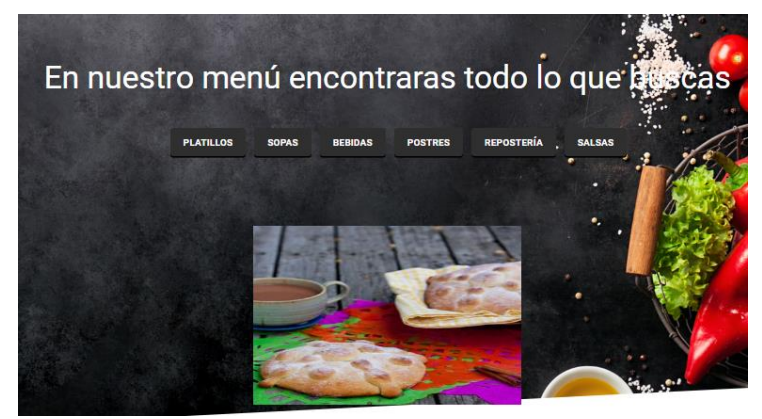

Figura 4 Pantalla del menú de recetas

Fuente: Elaboración Propia

BETANCOURT-SÁNCHEZ, Ricardo Gabino, LEVARIOTORRES, José Guadalupe, VILLANUEVA-FLORES, Juan Antonio y GALLEGOS-HERRERA, Juan Manuel. Software para administrar recetas de comida típica de la región. Revista de Tecnología Informática. 2019 
En la Imagen 5 se muestra como también se podrá encontrar recetas de temporada, todo esto en la página de inicio, podrá visualizar todo tipo de sugerencias que ya algunos usuarios han decidido publicar para quienes no estén registrados en la página.

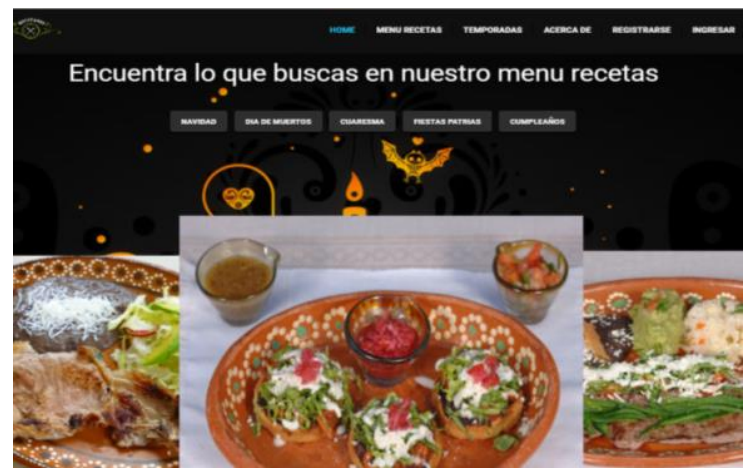

Figura 5 Página de inicio

Fuente: Elaboración Propia

En la Imagen 6 se muestra la pantalla que da la opción de que en caso de estar interesado en subir algún tipo de contenido tendrá la opción de registrarse y contará con otro tipo de privilegios en la página web.

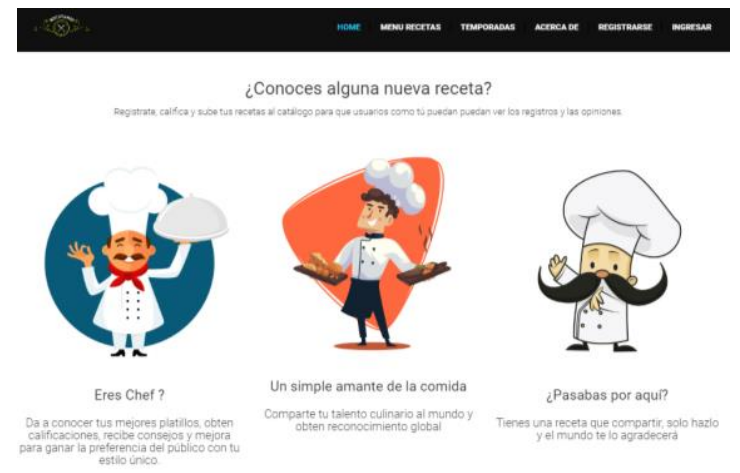

Figura 6 Pantalla de invitación a registro Fuente: Elaboración Propia

En la Imagen 7 se muestra el formulario de registro y al momento de tener llenar toda la información que pide el formulario y dar clic en el botón de registrar se habrá dado de alta como usuario del recetario web.

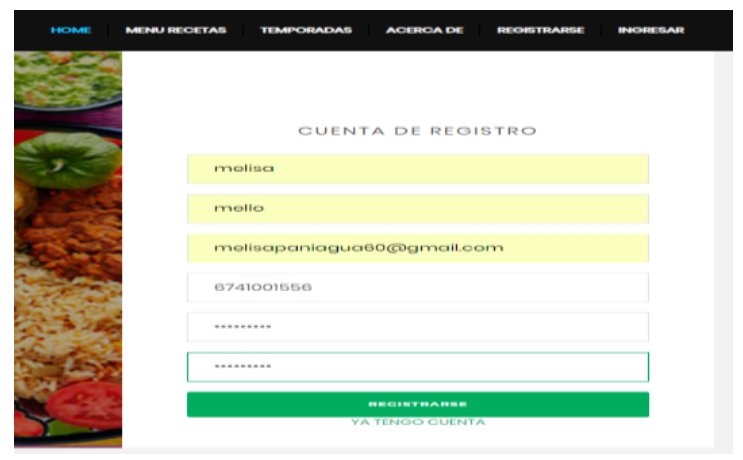

Figura 7 Pantalla de registro

ISSN 2531-2197

ECORFAN® Todos los derechos reservados
Después de quedar registrado deberá ir a la opción de ingresar el correo electrónico que dio y su contraseña para iniciar sesión, como lo muestra la Figura 8.

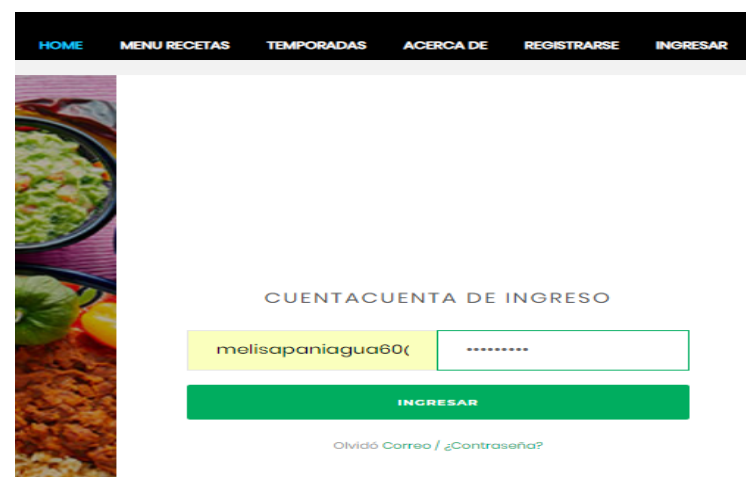

Figura 8 Pantalla de inicio de sesión Fuente: Elaboración Propia

La Figura 9 muestra que al contar con una cuenta de administrador, lo mandará al administrador donde tendrá privilegios como editar su usuario seleccionando la opción de usuarios, donde podrá agregar recetas y modificar todas las recetas que suba.

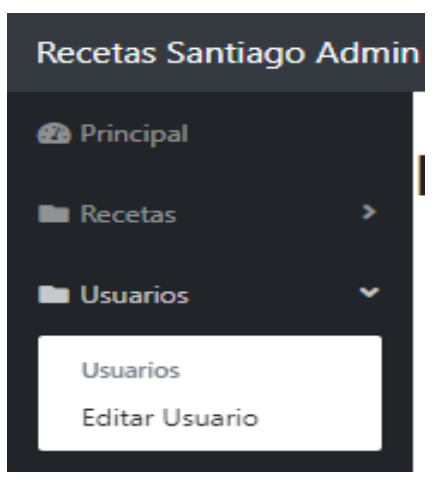

Figura 9 Pantalla de privilegios de administrador

La Figura 10 muestra la pantalla donde se puede editar la información de los usuarios.

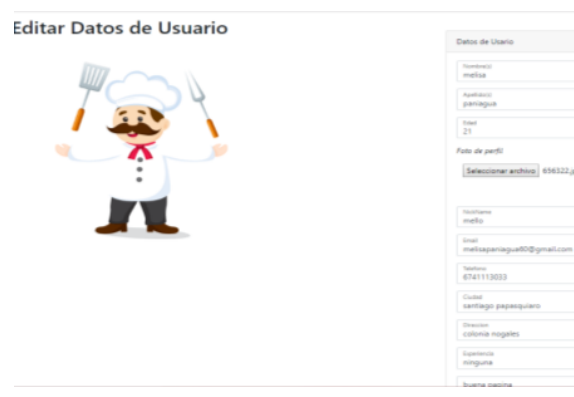

Figura 10 Pantalla de edición de datos de usuario Fuente: Elaboración Propia

Esta pantalla muestra los datos recabados previamente, los cuales puede modificar solamente un administrador, como ejemplo, se puede modificar nombre, apellidos, email, etc.

BETANCOURT-SÁNCHEZ， Ricardo Gabino, LEVARIOTORRES, José Guadalupe, VILLANUEVA-FLORES, Juan Antonio y GALLEGOS-HERRERA, Juan Manuel. Software para administrar recetas de comida típica de la región. Revista de Tecnología Informática. 2019 
Lo que se muestra en la Figura 11, es la pantalla que aparece en caso de seleccionar la opción de recetas, esa opción cuenta con los apartados de lista de recetas y agregar recetas donde podrá seleccionar cualquiera de las dos opciones.

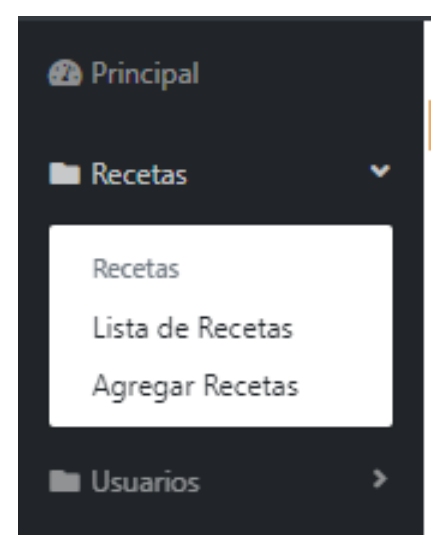

Figura 11 Pantalla de opciones a realizar con recetas Fuente: Elaboración Propia

Si decide ir a la opción de lista de recetas le aparecerá una tabla donde podrá ver todas las recetas que a ingresado, el nombre de la receta, categoría, región, el tiempo de realización, la fecha en que se subió, una subcategoría y de que temporada es. la Imagen 12 se muestra esta pantalla.

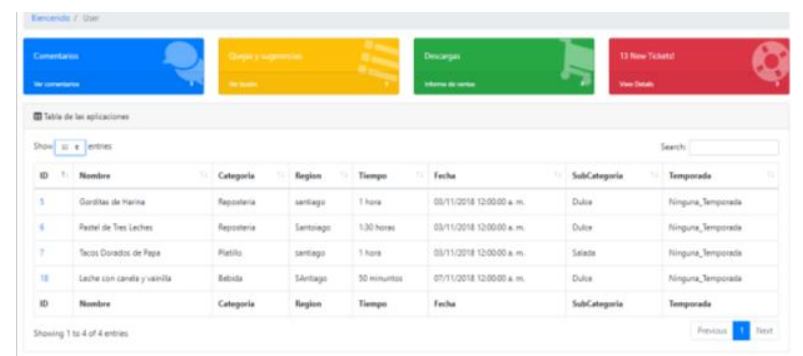

Figura 12 Pantalla de las propiedades de una receta. Fuente: Elaboración Propia

En la Imagen 13 aparece la pantalla de que en caso de contar con muchas recetas registradas y se busque una en especial podrá ver que se encuentra una opción de búsqueda donde ingresara el nombre de la receta para encontrarla más fácil.

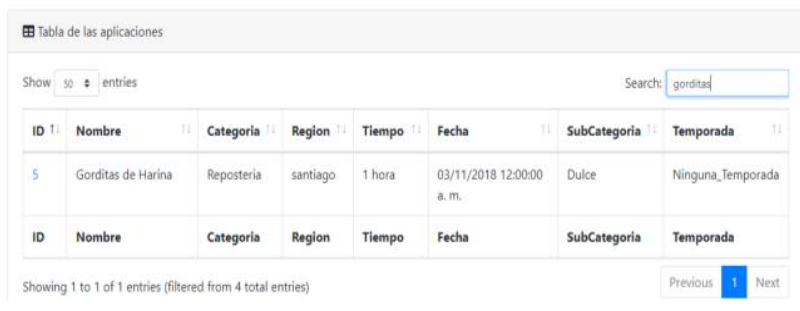

Figura 13 Pantalla de busqueda de receta específica Fuente: Elaboración Propia

ISSN 2531-2197

ECORFAN® Todos los derechos reservados
En esa misma página podrá observar como viene otras opciones donde podrá ver comentarios, quejas o sugerencias, descargas donde los demás usuarios o personas que no están registradas dejan acerca de cada una de las recetas que como usuario ha dejada registradas, lo cual se muetra en la Imagen 14.
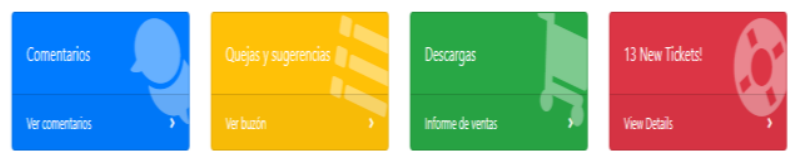

Figura 14 Secciones de comentarios y más Fuente: Elaboración Propia

Si se desea agregar una nueva receta deberá ir a esa opción donde al momento de dar clic aparecerá un formulario que deberá llenar con todos los datos de la receta. La Imágenes 15 muestra esta pantalla.

Registrar Nueva Receta
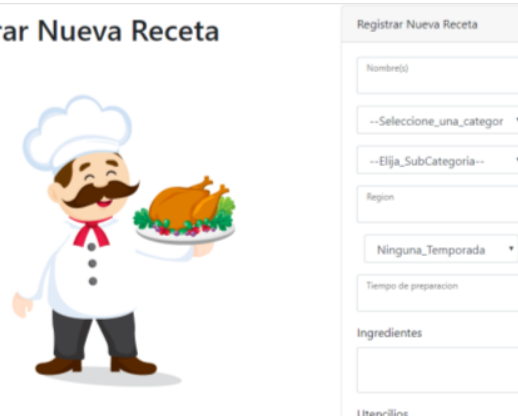

Figura 15 Pantalla para el registro de una nueva receta Fuente: Elaboración Propia

Es importante que al momento de llenar el formulario en el botón de seleccionar archivo deberá hacerlo por que en caso de no realizar este paso no se podrá guardar con éxito su receta, al momento de contar con todos los datos ingresados dará guardar a su receta para finalizar la operación.

\section{Agradecimiento}

Agradecemos al Instituto Tecnológico Superior de Santiago Papasquiaro y la ayuda de todos los compañeros que laboran como docentes en las carreras de Ingeniería en Sistemas Computacionales e Ingeniería en TIC's.

\section{Conclusiones}

El prototipo fue puesto a prueba con varios usuarios y el resultado fue de una gran aceptación tanto entre mujeres y hombres principalmente.

BETANCOURT-SÁNCHEZ, Ricardo Gabino, LEVARIOTORRES, José Guadalupe, VILLANUEVA-FLORES, Juan Antonio y GALLEGOS-HERRERA, Juan Manuel. Software para administrar recetas de comida típica de la región. Revista de Tecnología Informática. 2019 
En varios de los casos a los que fue sometido a prueba, no se dio una capacitación acerca del uso de las pantallas y su navegación, ya que se dejo a experimentación la adaptabilidad de su uso por parte de los ususarios, a lo que después de manipularlo, dichos usuarios comentaron que les fue muy sencillo el entender el funcionamiento del software.

Durante estas pruebas también se recabaron opiniones acerca de las posibles mejoras que se pudieran implementar para una siguiente fase de diseño, entre las cuales las más importantes fueron las de reducir la cantidad de botones para realizar las acciones.

Este prototipo puede mejorarse en un futuro considerando más aspectos de seguridad y protegerlo en cuanto a la manipulación de los niños que lo utilicen, en este rubro se podría cambiar el diseño de los botones, que les llame la atención a los niños, pero a la vez bloqueando los botones también para que no se exceda el manejo de los mismos.

En conclusión, se puede decir que el crear un software como este, representa todo un reto, ya que son proyectos que pueden crecer a lo que se desea, porque no se puede decir que tengan un término o tamaño óptimo de funcionalidad, pues son proyectos que se pueden ir adecuando cada vez más, e ir creciendo o diversificándose a medida o gusto de determinados clientes.

Consideramos que la aportación de conocimiento investigado e invertido por los involucrados, genera y aporta conocimiento en un tema de vital importancia en la actualidad.

Con respecto al apoyo que proporcionará la implementación de este software será de gran importancia para aquellas personas que se preocupen por llevar una alimentación más variada y con una conciencia de la preservación de la cultura y las tradiciones, siempre utilizando las tecnologías como un apoyo y beneficio.

\section{Referencias}

AGUILAR, L. J. (2010). Programacion en c/c++ java y UML. México: McGraw Hill.
BRAUDE Eric J. (2003), Ingeniería de Software una perspectiva orientada a objetos. México: Alfaomega.

DEITEL, D. y. (2010). Java Cómo Programar. México: Prentice Hall.

IESG, Instituto de Estudios Superiores en Gastronomía.

LAWRENCE, P. (1998). Como tratar la Resistencia al Cambio. Ltda. Colombia: Printer Colombiana.

PIATTINI M.G. (2007), Calidad de Sistemas Informáticos. México: Alfaomega.

PRESSMAN, R. S. (2010), Ingeniería del Software un enfoque práctico. México: MC Graw-Hill.

SOMMERVILLE, I. (2011), Ingeniería de Software. España: Pearson Addison Wesley

YÉNDEZ, N. V. (2004). La Inovación Tecnológica. MEDISAN.
BETANCOURT-SÁNCHEZ, Ricardo Gabino, LEVARIOTORRES, José Guadalupe, VILLANUEVA-FLORES, Juan Antonio y GALLEGOS-HERRERA, Juan Manuel. Software para administrar recetas de comida típica de la región. Revista de Tecnología Informática. 2019 